
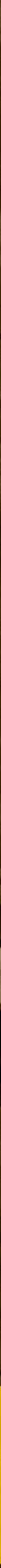

Claudia Marcela Castellanos Daza, Andrés David Gutiérrez Torres y Juan Gabriel Castañeda Polanco 


\title{
ACTITUDES HACIA LA DISCAPACIDAD EN EDUCACIÓN SUPERIOR
}

\section{ATTITUDES TOWARD DISABILITY IN HIGHER EDUCATION}

\author{
Claudia Marcela Castellanos Daza \\ ccastellanos@uniminuto.edu \\ Corporación Universitaria Minuto de Dios- UNIMINUTO \\ Cundinamarca-Colombia \\ Andrés David Gutiérrez Torres \\ andres.gutierrez@uniminuto.edu \\ Corporación Universitaria Minuto de Dios- UNIMINUTO \\ Cundinamarca-Colombia \\ Juan Gabriel Castañeda Polanco \\ jucastaneda@uniminuto.edu \\ Corporación Universitaria Minuto de Dios- UNIMINUTO \\ Cundinamarca-Colombia
}

\section{Resumen}

Objetivo. Identificar las actitudes de los profesores universitarios hacia la discapacidad.

Metodología. Estudio cuantitativo de tipo descriptivo y corte transversal, con un instrumento de actitudes (AIO) que midió acciones, intereses y opiniones relacionadas con la educación inclusiva. Se seleccionó una muestra de 70 profesores de la población total de la institución abordada, mediante muestreo no probabilístico por conveniencia.

Resultados. Los resultados sugieren que los profesores de mayor edad y mayor nivel de formación presentan una mejor actitud hacia la inclusión de sus estudiantes, por otro lado y aunque no existen diferencias significativas, las mujeres también presentan una tendencia positiva hacia las actitudes de inclusión; de igual forma el análisis descriptivo permite inferir que aún existen prejuicios que dificultan la educación inclusiva en las instituciones de educación superior.

Conclusión. Se espera que las conclusiones a las que se llegan en el presente estudio aporten elementos importantes para comprender la educación inclusiva en el marco actual de la educación superior en Colombia.

Palabras clave: educación Inclusiva, actitudes, discapacidad, necesidades educativas diversas. 


\section{Abstract}

Objective. To Identify the attitudes of university professors towards disability.

Methodology. A quantitative study of a descriptive and cross-sectional nature, with an attitudinal instrument (AIO) that measured actions, interests and opinions related to inclusive education. It was selected a sample of 70 teachers from the total population of the institution; using non-probabilistic sampling for convenience.

Results. The results suggest that older teachers and higher level of education have a better attitude towards the inclusion of their students, on the other hand, there are no significant differences, the women present a positive tendency towards inclusion attitudes; Similarly, the descriptive analysis allows us to infer that there are still prejudices that hinder inclusive education in higher education institutions.

Conclusion. It is expected that the conclusions reached in this study provide important elements to understand inclusive education in the current framework of higher education in Colombia.

Keywords: inclusive education, attitudes, disability, diverse educational needs. 


\section{INTRODUCCIÓN}

El ministerio de educación nacional de Colom-

Cbia en el año 2007, inicia su gestión en torno a los lineamientos de la educación inclusiva en universidades, gracias a estudios realizados con el centro de investigaciones para el desarrollo de la universidad nacional de Colombia, se evidenció que del total de instituciones de educativas superior (IES), 127 universidades (entre públicas y privadas) estaban comprometidas con la educación inclusiva.

Durante el proceso investigativo, el concepto de inclusión evolucionó en las instituciones de educación superior (IES); obedeciendo al nuevo concepto de discapacidad que propuso el convenio de Estocolmo de la organización de naciones unidas, en el que se concibió la discapacidad como una construcción social y no como una simple limitación del individuo; de tal manera que se vivió un cambio de paradigma en la atención a las personas con discapacidad, cambiando el término de estudiantes con necesidades educativas especiales (NEE), por el de Necesidades Educativas Diversas (NED); no obstante no fue sino hasta la publicación de la ley de discapacidad 1618 de 2013 , que el concepto tomó suficiente solidez como para ser parte fundamental de las políticas institucionales de las IES; sin embargo a la fecha no hay suficientes documentos que den cuenta de estrategias para los procesos de enseñanza- aprendizaje en el marco de la educación superior.

Es de suma importancia hacer la diferenciación entre los términos de inclusión educativa y de educación inclusiva, en el primer caso, se hace referencia a que el estudiante con una condición diversa se debe adaptar al sistema que se implementa en la institución de educación superior; y el segundo caso, se refiere estrictamente a la adaptación del sistema educativo en función de la diversidad del estudiante, de esta manera bajo este modelo se identifican las necesidades particulares del estudiante y las barreras del entorno que dificultan el acceso equitativo a sus derechos. así mismo, aparece en el camino el término de política de educación inclusiva, la cual hace referencia al conjunto de normas a nivel jurídico, (es decir, acciones, acuerdos, leyes, decretos, entre otros), que se desenvuelven en un ambiente de educación inclusiva con el fin de restituir derechos; en otras palabras, hace referencia al soporte legal del modelo de educación inclusiva (Opertti, 2008).
De esta forma, Velasquez, (2009) menciona que es preciso aclarar que el modelo de educación inclusiva no hace referencia a herramientas o un objetivo estratégico de las IES; sino justamente un modelo dinámico que se sustenta y aporta a la construcción de política pública; al ser dinámico, supone un cambio constante que al igual que el modelo actual de la ciencia, no se define como una verdad absoluta, sino como un proceso falseable sujeto a la discusión constante; de ahí la importancia de visibilizar experiencias significativas para generar nuevos conocimientos, nuevos debates, nuevas alternativas de restitución de derechos. Por otra parte, es importante comprender que la educación inclusiva implica otros términos relacionados, que son necesarios comprender para abordar el proceso con las poblaciones diversas, de una forma más completa, adecuada y pertinente, dentro de los cuales se encuentran: participación, diversidad, interculturalidad, equidad, pertinencia y calidad.

Para comprender el contexto conceptual que enmarca la educación inclusiva es necesario conocer los siguientes aspectos a la luz del decreto 1421 de 2017 expedido por el Ministerio de Educación Nacional de Colombia:

Accesibilidad: serie de acciones que garantizan la igualdad de condiciones en términos de acceso a ambientes físicos, virtuales, transporte, educación y comunicaciones. En este sentido se promueve la eliminación de barreras que impidan a la persona con discapacidad el acceso y el ejercicio pleno de sus derechos.

Acceso a la educación para las personas con discapacidad: proceso de creación de diversas estrategias que emplean las instituciones educativas para promover el ingreso a la educación de las personas con discapacidad, de manera que se garantice la equidad entre toda la comunidad educativa y se incentiva la no discriminación.

Acciones afirmativas: acciones encaminadas a disminuir las barreras culturales, sociales, económicas, de infraestructura, educacionales y actitudinales, con el fin de favorecer a la población con discapacidad para lograr la equidad social y educativa. (Véase Ley 1618 de 2013).

Ajustes razonables: acciones (tangibles o intangibles), estrategias, apoyos recursos o modificaciones pertinentes dentro del sistema educativo, teniendo en cuenta las necesidades parti- 
culares de los estudiantes con discapacidad. Con estas acciones se garantizan el aprendizaje, la participación, la evolución del proceso formativo y el pleno ejercicio de los derechos del estudiante con discapacidad.

Currículo flexible: diseño general, del plan de estudios, que toma en consideración la flexibilización metodológica, de manera que se brinden diferentes alternativas para acceder a la educación, adaptando los contenidos a las necesidades de los estudiantes con discapacidad en función de la diversidad social, cultural y estilos de aprendizaje. Es importante aclarar que en el currículo flexible los objetivos y la exigencia se mantienen iguales para todos los estudiantes.

Diseño universal del aprendizaje (DUA): hace referencia a los entornos, programas, currículos y servicios educativos inclusivos y accesibles con el fin de que la experiencia de aprendizaje sea significativa a partir del reconocimiento de la diversidad y la individualidad de cada estudiante. Este diseño permite transformar la práctica pedagógica del docente y facilita la evaluación de los aprendizajes.

Diversidad: característica inherente del ser humano; dentro de este concepto se busca la atención a todas las poblaciones que por razones sociales, económicas, políticas, culturales, lingüísticas, físicas, geográficas o psicosociales requieren protección de sus derechos. En este sentido se debe promover la visibilización y el respeto por la diversidad sin que ello afecte la intimidad de cada persona.

Equidad: respeto y reconocimiento de la diversidad de las personas. Un sistema educativo en el que sobresalga la equidad se caracteriza porque en él se ejecutan ajustes razonables adaptables a la diversidad en función de un enfoque diferencial, es decir una educación acorde a las diferencias y necesidades individuales, que tiene en cuenta factores sociales, económicos, políticos, culturales, físicos y geográficos.

El término también hace referencia a la accesibilidad, es decir que todas las personas puedan entender su entorno y acceder a la educación sin importar la condición en la que se encuentre.

Educación inclusiva: proceso educativo que responde de manera oportuna y adecuada a las características, los intereses, las expectativas y la diversidad de los estudiantes, con el fin de promover el aprendizaje y la participación en un ambiente libre de discriminación y barreras (tangibles e intangibles) que dificulten la educación; garantizando los apoyos, recursos y ajustes razonables necesarios para potencializar el aprendizaje.

Esquema de atención educativa: proceso en el que la institución educativa garantiza el ingreso, la permanencia y la graduación de los estudiantes con discapacidad, atendiendo oportunamente los requerimientos en términos de currículo, contenidos, metodologías, desempeño y calidad educativa.

Estudiante con discapacidad: aquel que presenta condiciones diversas en los aspectos mental, cognitivo, sensorial o físico, y que al interactuar con diferentes barreras actitudinales, culturales, discriminadoras, físicas, institucionales o curriculares no logra acceder plenamente al aprendizaje y la participación efectiva y equitativa en la sociedad.

Necesidades educativas especiales (NEE): se refieren a la patologización de la diversidad de la persona. Se suele asociar este término al modelo médico de la discapacidad, en el cual la condición diversa se relaciona con el daño fisiológico que se posee y por ende requiere atención médica.

Necesidades educativas diversas (NED): se refieren a la focalización de la población con con-

Tabla 1. Estadísticos de fiabilidad del instrumento según SPSS

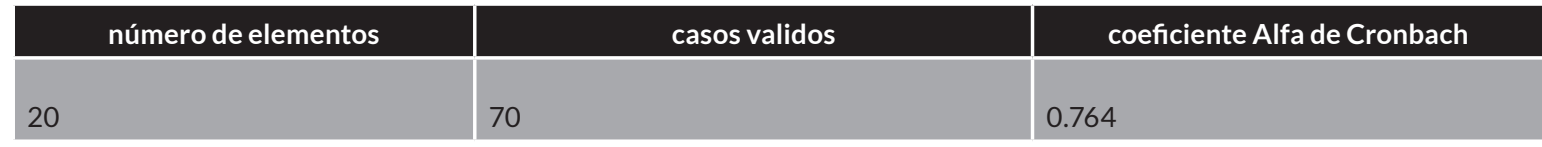

La tabla muestra el índice de confiabilidad y consistencia interna de la encuesta: actitudes hacia la discapacidad en educación superior 
diciones diversas a fin de examinar las barreras del sistema educativo en función de visibilizar la diversidad y fomentar su reconocimiento en la comunidad para así garantizar el pleno ejercicio de sus derechos.

Permanencia educativa para las personas con discapacidad: acciones, estrategias y servicios que ofrece la institución educativa con miras a fortalecer la adaptación a la vida universitaria y así facilitar, acompañar y hacer seguimiento en el proceso de permanencia y graduación a las personas con discapacidad. Estas acciones comprenden la promoción de los ajustes razonables en función de la pertinencia, eficacia, eficiencia y calidad del proceso educativo.

Núñez, Bienciento, Carpintero \& García (2014) afirman que dentro del proceso de la educación inclusiva existen varios factores para garantizar éxito en los procesos: trabajo interdisciplinar con el equipo docente, redes de apoyo con estudiantes en el aula, incorporación de tecnologías de información en la educación, flexibilización curricular y actitud positiva hacia la diversi- dad. Según el modelo de Rosenberg y Hovland (1960, citado por Llorens, Beas y Cifre, 2000), las actitudes pueden definirse como la intención conductual que tiene una persona en términos de tres dimensiones que comprenden factores de tipo afectivo, es decir la evaluación que hace la persona en término de agrado o desagrado; lo cognitivo, es decir creencias o ideas respecto a una situación, y, finalmente, el factor conductual, que hace referencia a la intención de emitir un comportamiento en particular en función de una situación específica. En ese orden de ideas, este constructo abarca tanto los estímulos que provocan la conducta como sus consecuencias, siempre con relación una situación o un proceso en particular.

De igual forma, para Fishbein y Ajzen (citado por Alonso, Navarro \& Vicente 2007), las actitudes se entienden como predisposiciones fruto del aprendizaje, las cuales se traducen en reacciones frente a personas, comunidades, objetos o situaciones. En ese sentido, el estudio de esta variable es fundamental para comprender las acciones y reacciones de las personas hacia la diversidad,

Tabla 2. Escala de actitudes hacia la discapacidad en educación superior.

\begin{tabular}{|c|c|c|c|c|c|}
\hline \multicolumn{2}{|c|}{ Estadístico } & \multirow{2}{*}{$\begin{array}{c}\text { Actitud positiva } \\
70\end{array}$} & \multirow{2}{*}{$\begin{array}{c}\text { Prejuicios } \\
70 \\
\end{array}$} & \multirow{2}{*}{$\begin{array}{c}\text { Educación } \\
\text { Inclusiva } \\
70\end{array}$} & \multirow{2}{*}{$\begin{array}{c}\text { Total AIO } \\
\\
70\end{array}$} \\
\hline N & Válido & & & & \\
\hline & Perdidos & 0 & 0 & 0 & 0 \\
\hline Media & & 21,90 & 18,59 & 22,29 & 62,77 \\
\hline Mediana & & 22,00 & 18,00 & 22,50 & 63,00 \\
\hline Moda & & 22 & 17 & 24 & 59 \\
\hline $\begin{array}{l}\text { Desviación } \\
\text { estándar }\end{array}$ & & 2,870 & 2,862 & 2,693 & 6,599 \\
\hline Mínimo & & 15 & 12 & 15 & 49 \\
\hline Máximo & & 28 & 24 & 28 & 80 \\
\hline Percentiles & 25 & 20,00 & 17,00 & 20,75 & 59,00 \\
\hline & 50 & 22,00 & 18,00 & 22,50 & 63,00 \\
\hline & 75 & 24,00 & 21,00 & 24,00 & 67,00 \\
\hline
\end{tabular}


específicamente las acciones de docentes de educación superior dirigidas hacia estudiantes con discapacidad.

Para comprender mejor el origen de las actitudes, Ajzen (citado por Muñoz, Novo \& Bellón, 2013) propone la teoría de la acción razonada. De acuerdo con esta, la actitud es una respuesta específica del individuo y no hace referencia a la totalidad de la conducta; para efectos del presente estudio, el término actitud designará las acciones concretas que los docentes llevan a cabo específicamente hacia las personas con discapacidad. De igual forma, se debe tener en cuenta el componente social; es decir, la influencia que tiene un grupo de personas, cultura o normas sociales, sobre la percepción del individuo frente a personas, grupos de personas o situaciones específicas. En el caso concreto de esta investigación, puede hacer referencia a los niveles sociales que rodean al individuo desde un modelo multinivel: contexto familiar, académico, educativo, laboral y su influencia en la forma en que dirige su conducta.

Tras la revisión del marco legal, conceptual y teórico se plantea como problema de investigación ¿Cuáles son las actitudes de los profesores universitarios hacia la discapacidad en una institución de educación superior?

\section{Metodología}

\section{Diseño}

Esta investigación es de enfoque cuantitativo, de tipo descriptivo con tendencia correlacional; este tipo de estudios correlaciónales, tiene como finalidad conocer la relación o grado de asociación que exista entre dos o más conceptos, categorías o variables en una muestra o contexto en particular (Hernández, Fernández y Baptista 2010 Pág.93).

\section{Objetivos}

\section{Objetivo General}

Identificar las actitudes de los profesores universitarios hacia las personas con discapacidad en la institución de educación superior.

\section{Objetivos específicos}

Establecer la relación que existe entre las actitudes hacia la discapacidad y las variables sociodemográficas de profesores universitarios.

Identificar las acciones inclusivas y no inclusivas de profesores de educación superior.

Tabla 3. Sexo y factores AIO

\begin{tabular}{|c|c|c|c|c|c|}
\hline \multicolumn{2}{|c|}{ Sexo/Encuesta AIO } & Actitud & Prejuicios & Educación & Total \\
\hline \multirow[t]{3}{*}{ Mujeres } & Media & 22,88 & 19,61 & 22,88 & 65,36 \\
\hline & $\mathrm{N}$ & 33 & 33 & 33 & 33 \\
\hline & $\begin{array}{l}\text { Desviación } \\
\text { estándar }\end{array}$ & 2,666 & 2,794 & 2,655 & 5,857 \\
\hline \multirow[t]{3}{*}{ Hombres } & Media & 21,03 & 17,68 & 21,76 & 60,46 \\
\hline & $\mathrm{N}$ & 37 & 37 & 37 & 37 \\
\hline & $\begin{array}{l}\text { Desviación } \\
\text { estándar }\end{array}$ & 2,794 & 2,636 & 2,650 & 6,427 \\
\hline \multirow[t]{3}{*}{ Total } & Media & 21,90 & 18,59 & 22,29 & 62,77 \\
\hline & $\mathrm{N}$ & 70 & 70 & 70 & 70 \\
\hline & $\begin{array}{l}\text { Desviación } \\
\text { estándar }\end{array}$ & 2,870 & 2,862 & 2,693 & 6,599 \\
\hline
\end{tabular}


Tabla 4. Nivel de formación y factores AIO

\begin{tabular}{|c|c|c|c|c|c|}
\hline \multicolumn{2}{|c|}{ Nivel de estudios/Escala } & \multirow{2}{*}{$\begin{array}{c}\text { Actitud } \\
20,00\end{array}$} & \multirow{2}{*}{$\begin{array}{c}\text { Prejuicios } \\
16,00\end{array}$} & \multirow{2}{*}{$\begin{array}{c}\begin{array}{c}\text { Educación } \\
\text { Inclusiva }\end{array} \\
23,00\end{array}$} & \multirow{2}{*}{$\begin{array}{l}\text { total } \\
59,00\end{array}$} \\
\hline \multirow{3}{*}{ Tecnólogo } & Media & & & & \\
\hline & $\mathrm{N}$ & 1 & 1 & 1 & 1 \\
\hline & $\begin{array}{l}\text { Desviación } \\
\text { estándar }\end{array}$ & & & & \\
\hline \multirow{3}{*}{ Profesional } & Media & 20,89 & 19,39 & 22,11 & 62,39 \\
\hline & $\mathrm{N}$ & 18 & 18 & 18 & 18 \\
\hline & $\begin{array}{l}\text { Desviación } \\
\text { estándar }\end{array}$ & 3,085 & 2,524 & 2,698 & 6,608 \\
\hline \multirow{3}{*}{ Especialización } & Media & 21,70 & 18,04 & 22,11 & 61,85 \\
\hline & $\mathrm{N}$ & 27 & 27 & 27 & 27 \\
\hline & $\begin{array}{l}\text { Desviación } \\
\text { estándar }\end{array}$ & 2,267 & 2,849 & 1,928 & 5,675 \\
\hline \multirow{3}{*}{ Maestría } & Media & 22,78 & 18,48 & 22,57 & 63,83 \\
\hline & $\mathrm{N}$ & 23 & 23 & 23 & 23 \\
\hline & $\begin{array}{l}\text { Desviación } \\
\text { estándar }\end{array}$ & 3,059 & 2,937 & 3,540 & 7,487 \\
\hline \multirow{3}{*}{ Doctorado } & Media & 27,00 & 24,00 & 23,00 & 74,00 \\
\hline & $\mathrm{N}$ & 1 & 1 & 1 & 1 \\
\hline & $\begin{array}{l}\text { Desviación } \\
\text { estándar }\end{array}$ & & & & \\
\hline \multirow{2}{*}{ Total } & Media & 21,90 & 18,59 & 22,29 & 62,77 \\
\hline & $\mathrm{N}$ & 70 & 70 & 70 & 70 \\
\hline
\end{tabular}

Identificar las opiniones y/o prejuicios de los profesores de educación superior acerca de la discapacidad.

\section{Población}

Se obtiene una muestra no probabilística intencionada de 70 profesores universitarios de un total de 297 participantes que presentan un promedio de edad de 38 años de edad. El 47.1\% de los participantes eran mujeres (33) y el 52.9 (37) de los participantes eran hombres, dentro de los criterios de inclusión se tuvo en cuenta los años de experiencia docente (promedio 8) y el nivel educativo de los profesores, de los cuales 1 tenía nivel de formación de tecnólogo, 18 profesionales, 27 con especialización, 23 con maestría y 1 con doctorado. Dentro de los criterios de exclusión fue el no estar ejerciendo la docencia y el tiempo de experiencia. 
Tabla 5. Experiencia estudiantes con discapacidad y factores AIO

\begin{tabular}{|c|c|c|c|c|c|}
\hline \multicolumn{2}{|c|}{ Estudiantes con discapacidad/Escala } & \multirow{2}{*}{$\begin{array}{c}\text { Actitud } \\
21,46\end{array}$} & \multirow{2}{*}{$\begin{array}{c}\text { Prejuicios } \\
18,07\end{array}$} & \multirow{2}{*}{$\begin{array}{c}\text { Educación } \\
\text { Inclusiva }\end{array}$} & \multirow{2}{*}{$\begin{array}{l}\text { total } \\
61,57\end{array}$} \\
\hline NO & Media & & & & \\
\hline & $\mathrm{N}$ & 28 & 28 & 28 & 28 \\
\hline & $\begin{array}{l}\text { Desviación } \\
\text { estándar }\end{array}$ & 2,687 & 2,227 & 2,912 & 6,385 \\
\hline \multirow[t]{3}{*}{ SI } & Media & 22,19 & 18,93 & 22,45 & 63,57 \\
\hline & $\mathrm{N}$ & 42 & 42 & 42 & 42 \\
\hline & $\begin{array}{l}\text { Desviación } \\
\text { estándar }\end{array}$ & 2,982 & 3,196 & 2,559 & 6,692 \\
\hline \multirow[t]{3}{*}{ Total } & Media & 21,90 & 18,59 & 22,29 & 62,77 \\
\hline & $\mathrm{N}$ & 70 & 70 & 70 & 70 \\
\hline & $\begin{array}{l}\text { Desviación } \\
\text { estándar }\end{array}$ & 2,870 & 2,862 & 2,693 & 6,599 \\
\hline
\end{tabular}

\section{Instrumentos}

Se diseñó una encuesta de actitudes intereses y opiniones (AIO) de 20 ítems en escala likert; la cual midió el constructo actitudes hacia la discapacidad en educación superior; en una escala ordinal de 1 a 4: donde 1 significa muy en desacuerdo; 2 significa desacuerdo; 3 indica de acuerdo y 4 significa totalmente de acuerdo. La encuesta fue diseñada con tres categorías que también fueron tomadas en cuenta para la sistematización: actitud positiva (acciones encaminadas a la inclusión), prejuicios (percepciones y emociones respecto a las personas con discapacidad) y educación inclusiva (conocimiento del concepto en el quehacer docente). Para medir la consistencia interna del instrumento y la confiabilidad de la encuesta, se sometió a la prueba, al análisis estadístico del coeficiente Alfa de Cronbach, mediante el paquete estadístico SPSS 22, el valor obtenido en dicho coeficiente es de 0.764 el cual indica que el instrumento presenta consistencia interna.

\section{Procedimiento}

Este trabajo de investigación se llevó a cabo en las siguientes fases:

Fase 1 construcción de la parte Teórica: se consolida los objetivos, justificación, introduc- ción, marco teórico, marco referencial y marco legal del proyecto de investigación.

Fase 2 diseño y validación del instrumento: se realiza la validación mediante estadístico alfa de Cronbach, validación de jueces y prueba cognitiva.

Fase 3 aplicaciones del Cuestionario AIO: se ejecuta la implementación del cuestionario que mide las actitudes hacia la discapacidad en educación superior.

Fase 4 Análisis y Tabulación: al obtener los resultados del cuestionario AIO se realiza su respectivo análisis de confiabilidad y análisis estadístico en el programa SPSS 22 que ayudara a la posterior interpretación de resultados.

Fase 5 Conclusiones: de acuerdo a los resultados obtenidos se hace el análisis pertinente de estos para así llegar a conclusiones predictivas para la investigación.

\section{Resultados}

Para medir la consistencia interna del instrumento y la confiabilidad de la encuesta, se sometió a la prueba, al análisis estadístico del coeficiente alfa de Cronbach, mediante el paquete estadístico SPSS; cómo se puede observar en 
la tabla 1. el valor obtenido en dicho coeficiente es de 0.738

Tal como lo indica la tabla 1 el valor del coeficiente alfa de Cronbach, indica valores aceptables de confiabilidad; lo cual quiere decir, que el instrumento tiene consistencia interna que permite medir el constructo "actitud" de manera fiable; sin embargo, se recomienda hacer una reformulación en algunas preguntas; para que el coeficiente alfa pueda aumentar y los resultados sean más consistentes. Cabe resaltar que según Oviedo Y Campo (2005) Y Hernandez, Fernandez \& Baptista (2006); el valor mínimo aceptable del coeficiente alfa es de 0.70; considerándose los valores inferiores como poco confiables. tal como lo plantea Bojorquez, Lopez, Hernandez \& Jiménez (2013): "un valor superior a 0.7 revela una fuerte relación entre las preguntas, un valor inferior revela una débil relación entre ellas"

De esta forma, al aplicar la encuesta de actitudes, intereses y opiniones (AIO) de 20 ítems el cual midió el constructo de actitudes hacia la discapacidad en educación superior. En la tabla 1 se evidencian las tres categorías que fueron tomadas en cuenta para la sistematización: actitud positiva, prejuicios y educación inclusiva.

La puntuación mínima de los encuestados fue de 49 puntos en la prueba AIO, mientras que el máximo es de 80 puntos, el $25 \%$ de los participantes presentaron una puntuación menor a 59, lo que demuestra que percibieron de una forma con mayor tendencia al desacuerdo hacia las actitudes, intereses y opiniones de la inclusión, mientras que otro $25 \%$ presentaron puntuaciones por encima de 67 que demuestran percibir que estas totalmente de acuerdo con las actitudes hacia la inclusión.

Los participantes demuestran interés por adelantar procesos inclusivos con personas con discapacidad en el marco de la educación superior; sin embargo, el 17 \% (12 personas encuestadas) prefieren no tener ningún estudiante con discapacidad en sus aulas de clase. Esto indica que, aunque sea en una proporción baja, aún se presentan pensamientos discriminatorios que pueden dificultar los procesos inclusivos en las instituciones educativas de educación superior.

En la tabla 3 se puede evidenciar que no existen diferencias significativas en la percepción que tienen los profesores hombres o mujeres hacia las actitudes de inclusión, sin embargo los hombres en relación a los perjuicios se encuentran por debajo de la media al igual que en la percepción total de la inclusión, es decir que aunque no existe diferencia estadísticamente significativa, los hombre si presentar una mayor tendencia a estar en desacuerdo con las actitudes de inclusión.

El nivel de formación de los profesores evidencia un factor de calidad en la educación universitaria, en la tabla 4 se evidencia que entre mayor sea el nivel de formación del profesor se presenta una mejor percepción o actitud hacia la inclusión. A pesar de que no existen diferencias

Tabla 6. Correlación de Pearson entre edad, años de experiencia docente y factores AIO

\begin{tabular}{|c|c|c|c|c|c|}
\hline \multicolumn{2}{|c|}{ Correlaciones } & \multirow{2}{*}{$\begin{array}{c}\text { Actitud } \\
\text {,142 }\end{array}$} & \multirow{2}{*}{$\begin{array}{c}\text { Prejuicios } \\
\text {,246* }\end{array}$} & \multirow{2}{*}{$\begin{array}{c}\text { Educación } \\
\text { Inclusiva } \\
\text {,075 }\end{array}$} & \multirow{2}{*}{$\begin{array}{l}\text { total } \\
\text {,205 }\end{array}$} \\
\hline Edad & $\begin{array}{l}\text { Coeficiente de } \\
\text { correlación }\end{array}$ & & & & \\
\hline & Sig. (bilateral) & ,241 & ,040 &, 535 & ,089 \\
\hline & $\mathrm{N}$ & 70 & 70 & 70 & 70 \\
\hline \multirow[t]{3}{*}{$\begin{array}{l}\text { Años de experiencia } \\
\text { docente }\end{array}$} & $\begin{array}{l}\text { Coeficiente de } \\
\text { correlación }\end{array}$ & ,118 &,- 023 & ,122 & ,106 \\
\hline & Sig. (bilateral) & ,332 & ,847 & ,316 & ,385 \\
\hline & $\mathrm{N}$ & 70 & 70 & 70 & 70 \\
\hline
\end{tabular}

*. La correlación es significativa en el nivel 0,05 ( 2 colas). 
significativas en cada factor de la prueba en las puntuaciones generales si se percibe una tendencia positiva por el nivel de formación.

La experiencia de tener estudiantes con algún tipo de discapacidad se esperaría que sensibilizará a los profesores universitarios frente a su actitud hacia la inclusión, sin embargo como se evidencia en la tabla 5, no existen diferencias significativas entre los profesores que han tenido estudiantes con discapacidad y los que no.

En la tabla 6 se observa que existe una correlación positiva entre la edad y los prejuicios, es decir que entre mayor sea la edad del profesor, se tiene una mayor actitud hacia la inclusión, mientras que entre más joven es el profesor se percibe como estar más desacuerdo con las actitudes de inclusión.

Por otro lado, dentro del análisis descriptivo sobresalen los puntajes obtenidos en la encuesta a la pregunta "¿estaría dispuesto a modificar su metodología de clase por la presencia de un estudiante con discapacidad en su clase?" Los resultados arrojan que el $81 \%$ de la muestra abordada no modificaría su metodología, lo cual supone un obstáculo en los procesos de educación inclusiva en educación superior. De igual forma, se encontró que el $73 \%$ de los docentes abordados consideran que deberían existir docentes e instituciones especializadas para la educación de personas con discapacidad; esto es significativo ya que el enfoque actual de educación inclusiva dominada por el ministerio de educación nacional, plantea que es el sistema educativo el que debe adecuarse a las necesidades del estudiante diverso y no al revés. (ley 1618 de 2013). Finalmente se encuentra que uno de lo resultados más significativos de este estudio descriptivo radica en la pregunta "considera que las IES deberían rechazar el ingreso de personas con discapacidad" a la cual el $76 \%$ de la muestra aceptó la afirmación, lo cual supone un prejuicio que impide la ejecución efectiva y necesaria de la educación inclusiva en la educación superior. Cabe resaltar que sólo 17 docentes (24\%) manifiesta rechazo frente a la premisa; lo cual se relaciona con un concepto más acorde a una institución inclusiva.

\section{Conclusiones}

Tras los objetivos planteados en el estudio, se puede evidenciar que los profesores mayor edad y mayor nivel de formación presentan una mejor actitud hacia la inclusión de sus estudiantes, por otro lado aunque no existen diferencias significativas, las mujeres también presentan una tendencia positiva hacia las actitudes de inclusión.

Del análisis descriptivo de resultados se puede concluir que aún hay docentes que se muestran renuentes a la posibilidad de brindar clases a las personas con discapacidad, lo cual se relaciona con lo propuesto por Hergreaves (2005), acerca de la vida emocional del docente, partiendo de las concepciones, sentimientos y percepciones del profesor en el ámbito académico que se desenvuelve.

Otro resultado importante para compartir en este estudio, es que si el docente tiene experiencia o manejo con personas que tienen discapacidad, no estarían dispuestos a compartir sus experiencias con otros profesores.

A partir de lo anterior, se realizan las siguientes recomendaciones tras la revisión de las estrategias de inclusión para las instituciones de educación superior:

- Establecer alianzas institucionales expertas en el tema que permitan la construcción de nuevos conocimientos respecto a la educación inclusiva en la IES

- Generar grupos y semilleros de investigación con el fin de que la IES se contextualice en términos de la educación inclusiva.

- Incluir en los planes de trabajo profesoral horas destinadas para el apoyo docente a la educación inclusiva, de manera que se conviertan en un referente o promotor del tema en el programa académico.

- Espacios de capacitación y cualificación a personal administrativo (admisiones, registro y control, tesorería, facturación, personal de seguridad y aseo, decanaturas y demás colaboradores de la IES) en función de brindar un servicio al cliente de manera inclusiva.

- Adaptación y flexibilización de currículos y metodologías de enseñanza-aprendizaje que se adapten a las condiciones diversas de los estudiantes, de una manera didáctica, innovadora y equitativa.

- Ofrecer a los estudiantes servicios de apoyo académico y psicosocial para el fomento de 
la educación inclusiva y la permanencia de la población con necesidades educativas diversas.

- Articular los lineamientos de la educación inclusiva con el Proyecto Educativo Institucional, de manera que los principios institucionales, así como la misión y la visión promuevan la inclusión de la población diversa.

- La institución educativa debe generar espacios de cualificación para el fomento de la educación inclusiva y el reconocimiento a los docentes que tienen competencias inclusivas y que las desarrollan con éxito en su práctica profesional. Esta estrategia permitiría construir una cultura inclusiva institucional.

- Generar espacios académicos de socialización, análisis y evolución constante de los procesos inclusivos en el contexto de la educación superior 


\section{Referencias bibliográficas}

Alonso, M., Navarro, R., \& Vicente, L. (2007). Actitudes hacia la diversidad en estudiantes universitarios. Recuperado de: http://repositori.uji.es/xmlui/bitstream/handle/10234/78529/forum_2007_46.pdf?sequence=1

Avalos, B. y Cavada, P. (2010), la profesión docente: temas y discusiones en la literatura internacional. Estudios Pedagógicos, 36 (1), 235-263. http://dx.doi.org/10.4067/So718-07052010000100013

Blanco, R. (2008) Marco conceptual de educación inclusiva (Inclusión educativa: el camino del futuro). Recuperado de: http://www.ibe.unesco.org/fileadmin/user_upload/Policy_Dialogue/48th_ICE/CONFINTED_48_Inf_2__Spanish.pdf

Bójorquez, J., López, L., Hernández, M., \& Jiménez, E. (2013). Utilización del alfa de Cronbach para validar la confiabilidad de un instumento de medición de satisfacción del estudiante en el uso de Software Minitab. Innovation in Engineering, Technology and Education for Competitiveness and Prosperity, 1-9.

Constitución Política de Colombia. Bogotá, Colombia. 1991. Recuperado de: http://www.procuraduria.gov.co/guiamp/ media/file/Macroproceso\%2oDisciplinario/Constitucion_Politica_de_Colombia.htm

Corporación Universitaria Minuto de Dios. (2014) Proyecto educativo institucional Uniminuto. Schristian Supelano

Fernández JA, Hernández R, Siegrist J. (2001). El perfil de calidad de vida para enfermos crónicos (PECVEC): un método para evaluar bienestar y funcionalismo en la práctica clínica. Atención Primaria. 28: 680-9.

Freire, A. Linhalis, F. Bianchini, S. Fortes, R Graça, M. \& Pimentel, C (2009) Revealing the whiteboard to blind students: An inclusive approach to provide mediation in synchronous e-learning activities. Computers \& Education. 54 (4), 866 - 876. http://dx.doi.org.ezproxy.uniminuto.edu:8000/10.1016/j.compedu.2009.09.016

González, J. \& Baños, L. (2012) Estudio sobre el cambio de actitudes hacia la discapacidad en clases de actividad física. Recuperado de: http://scielo.isciii.es/pdf/cpd/v12n2/articulo1o.pdf

Gómez-Torres, F. (2015) Los sentimientos y las emociones en la identidad profesional del profesor. Praxis pedagógica 16,3952.Recuperado de: http://biblioteca.uniminuto.edu/ojs/index.php/praxis/article/view/1222/1198

Hernández, R. Fernández, C. y Baptista, P. (2006) Metodología de la investigación. Recuperado de: https://competenciashg. files.wordpress.com/2012/10/sampieri-et-al-metodologia-de-la-investigacion-4ta-edicion-sampieri-2006_ocr.pdf

Hargreaves,A. (2005), Profesorado, cultura y posmodernidad. Madrid: Morata

Jimenez, R. (2002) Las personas con discapacidad en la educación superior. Recuperado de: http://portal.uc3m.es/portal/ page/portal/cultura_y_deporte/discapacidad/recursos/documentos/educacion/Disceneducsuperior.pdf

Instituto Nacional para Ciegos (2015) Nuestra historia. Recuperado de http://www.inci.gov.co/acerca-del-inci/nuestrahistoria

Lazarus R, (2000). Estrés y emoción. Manejo e implicaciones en nuestra salud. España: Bilbao.

Llorens, S. Beas, M. y Cifre, E. (2000) Diseño y validación de un instrumento de evaluación de actitudes hacia la búsqueda de empleo (A.B.E.). Recuperado de: http://repositori.uji.es/xmlui/bitstream/handle/10234/80067/Forum_1999_15. pdf?sequence $=$

MEN (2013) Lineamientos Politicas de educación superior inclusiva. Recuperado de: http://redes.colombiaaprende.edu.co/ ntg/men/pdf/Lineamientos.pdf

Ministerio de Telecomunicaciones (2015) Ministerio tic alfabetiza digitalmente a personas ciegas y con baja visión del país. Recuperado de http://micrositios.mintic.gov.co/convertic/

Muñoz, J., Novo, I. \& Bellón, E. (2013) La inclusión de los estudiantes universitarios con discapacidad en las universidades presenciales: actitudes e intención de apoyo por parte de sus compañeros. Recuperado de: https://www.unav.edu/ publicaciones/revistas/index.php/estudios-sobre-educacion/article/download/2026/1891

Nuñez, M. Bienciento, C. Carpintero, E. \& García, M. (2014) Enfoques de atención a la diversidad, estrategias de aprendizaje y motivación en educación secundaria. Perfiles educativos, 36 (145), 65-80. Http://dx.doi.org.ezproxy.uniminuto. edu:80oo/10.1016/s0185-2698(14)70638-5

Pastor, C. Sanchez, J. y Zubilaga A. (2015), Diseño Universal para el Aprendizaje (DUA) Pautas para su introducción en el currículo. Recuperado de http://www.educadua.es/doc/dua/dua_pautas_intro_cv.pdf 
Opertti, R. (2008) Inclusión educativa: el camino del futuro. Un desafío para compartir. Recuperado de:http://www.ibe. unesco.org/fileadmin/user_upload/COPs/News_documents/2007/0710PanamaCity/Documento_Inclusion_Educativa. pdf

Osorno, M. Sanabria, L. Ramirez, I. Duvian, F. y Barón, C. (2012) Pautas para la interacción. Recuperado de: http://www. colombiaaprende.edu.co/html/micrositios/1752/articles-325528_pautas.pdf

Oviedo, H. \& Campo, A. Aproximación al uso del coeficiente alfa de Cronbach.Recuperado de: http://www.scielo.org.co/ scielo.php?script=sci_arttext\&pid=So034-74502005000400009

Ruiz, L. (2001) La sistematización de prácticas. Recuperado de http://www.oei.es/equidad/liceo.PDF

Saez, R. (2006) La educación Intercultural. Recuperado de: http://www.revistaeducacion.mec.es/re339/re339a37.pdf

Velasquez, R. (2009) Hacia una nueva definición del concepto “política pública”. Recuperado de: http://www.ins.gov.co/ investigacion/docs/Hacia\%20una\%2onueva\%2odefinici\%C3\%B3n\%20de\%2opol\%C3\%ADtica\%2op\%C3\%BAblica.pdf

Vojtech, R. (2015) mathematics in inclusive education of blind students in secondary schools in the czech republic. Procedia social and behavioral sciences, 174 (12), 3933-3939. Doi:10.1016/j.sbspro.2015.01.1136

Yupanchi, A. Aranda, C. Vásquez, C. \& Verdugo, W. ( 2014) educación inclusiva y discapacidad: su incorporación en la formación profesional de la educación superior. Revista de educación superior, 43 (171), 93 -115. Http://dx.doi.org. ezproxy.uniminuto.edu:800o/10.1016/j.resu.2014.06.003

Zembylas, M. (2003). Interrogating "teacher identity": emotion, resistance, and self-formation. Educational Theory,53 (1) p.107. DOI: $10.1111 / \mathrm{j} .1741-5446.2003 .00107 \cdot \mathrm{x}$ 
Revista incluida en los siguientes agregadores de contenidos

Revista incluida en los siguientes directorios

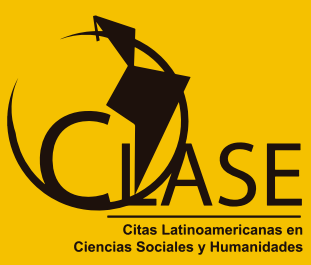

แlbiblat

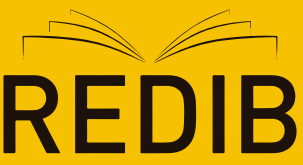

Red Iberoamericana

de Innovación y Conocimiento Científico

\section{Google Académico}

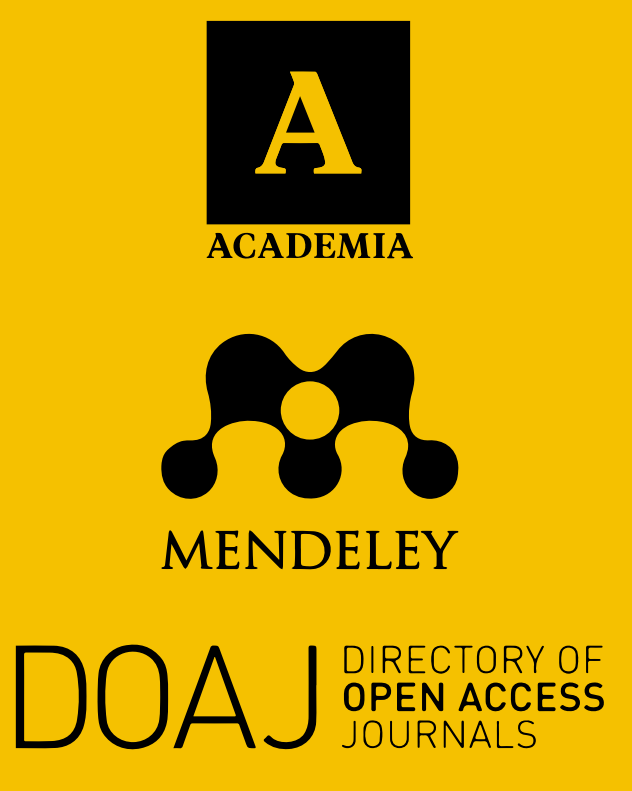

Revista incluida en la siguiente red social

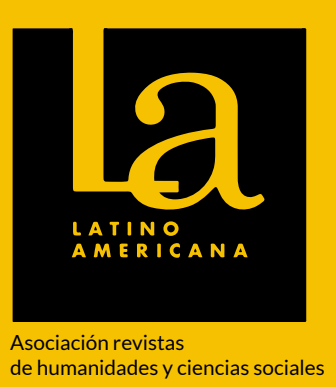



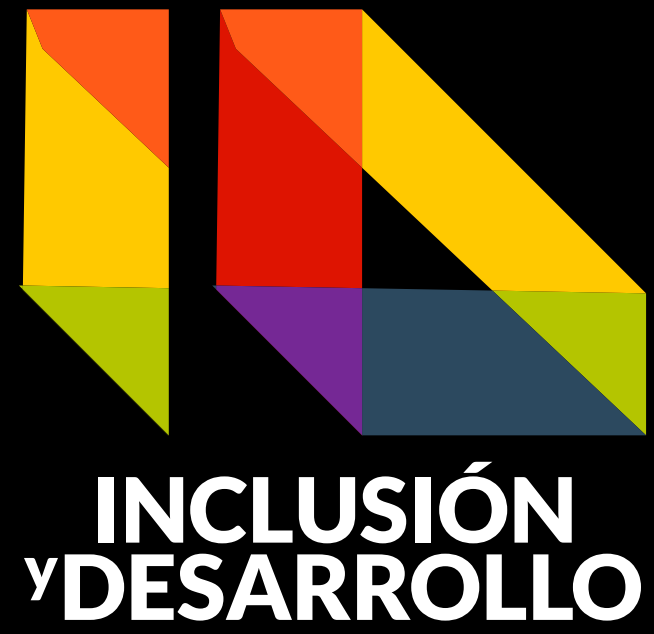

No. 2 Vol. 5 Año 2018 ISSN En línea: 2590-7700

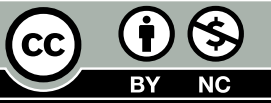

